QUADERNS DE FILOSOFIA VOL. V NÚM. I (20I 8): I I 5-I 26

eISSN: $234 \mathrm{I}-3042$ DOI: IO.7203/QFIA.5.I.I 2476

PABLO RYCHTER

Universitat de València

\title{
Rescatando los casos Mele/Robb
}

Resumen: Mi intención en este trabajo es exponer algunas dudas sobre las consideraciones que Carlos Moya (2017) esgrime en contra de un tipo particular de "Caso Frankfurt": el "caso de bloqueo" descrito por Mele y Robb (1998). En primer lugar, argumento que el caso de Mele/Robb es un contraejemplo exitoso al Principio de Posibilidades Alternativas aún si, en contra de lo que sus proponentes sugieren, la acción relevante está causada de manera determinista. Por otro lado, argumento que el agente en el caso Mele/Robb ejercita un tipo relevante de control racional sobre su acción.

Abstract: My goal in this paper is to expose some doubts about the arguments that Carlos Moya (2017) addresses against a particular Frankfurt-style case: the "blockage case" described by Mele and Robb (1998). I argue first that the Mele/ Robb case is a succesful counterexample to the Alternative Possibilities Principle even if, against what its proponents suggest, the relevant action is deterministically caused. Second, I argue that the agent in the Mele/Robb case exercises a relevant sort of rational control over her action.

Palabras clave: Frankfurt, Moya, sobredeterminación, sensibilidad a razones. Keywords: Frankfurt, Moya, overdetermination, reasons-responsiveness. 


\section{INTRODUCCIÓN}

$\mathrm{E}$ N El libre albedrio Carlos Moya argumenta que el llamado "control plural" es efectivamente, y no solo aparentemente, un componente constitutivo del libre albedrío y la responsabilidad moral. Es decir, argumenta en favor del Principio de Posibilidades Alternativas (PPA), el principio según el cual "una persona es moralmente responsable de lo que ha hecho solo si podría haber hecho algo distinto" (FrankfurT I 969, 829). El argumento supone una pormenorizada evaluación de las distintas variedades de "casos Frankfurt" (CF), los cuales son típicamente presentados como contraejemplos a PPA. Mi intención en este trabajo es exponer algunas dudas sobre las consideraciones que Moya esgrime en contra de un tipo particular de CF: los casos descritos por Mele y Robb (I 998), los cuales caen dentro de lo que Moya llama "casos de bloqueo". Si mis dudas están bien fundadas, no parece haber razones para negar que casos de este tipo sean contraejemplos genuinos al PPA, lo cual inclina la balanza en contra de la idea de que el control plural sea realmente una condición para el libre albedrío.

\section{Los casos Frankfurt, La objeción del dilema y EL CASO DE MELE/RobB}

Para llegar al punto que me interesa es necesario recorrer, aunque sea muy rápidamente, un transitado camino que discurre por CFs más o menos corrientes y por objeciones a estos también familiares. En los CFs clásicos nos encontramos con un agente, Jones, que lleva a cabo una acción $a$ como resultado de su propio razonamiento y decisión. ${ }^{1}$ Además, y sin que este hecho sea conocido por Jones, hay un segundo agente, Black, que observa el proceso de deliberación de Jones que culmina en $a$. Black está interesado en que Jones lleve a cabo $a$, de manera que si Black pensara, sobre la base de su observación del proceso deliberativo de Jones, que Jones no llevará a cabo $a$, entonces intervendría (tal vez utilizando una sofisticada tecnología proporcionada por la neurociencia) para hacer que Jones lleve a cabo $a$. Sin embargo, Black prefiere

\footnotetext{
${ }^{1}$ Podemos suponer aquí que el dominio relevante de $a$ incluye acciones ordinarias que involucran movimientos corporales (como disparar un arma), "acciones mentales" (como decidir disparar un arma), e incluso omisiones (como no disparar un arma). Pueden construirse CFs con los tres supuestos. El CF original de Frankfurt (1969) parece involucrar acciones ordinarias, pero discusiones posteriores (entre ellas, la de Moya) se centran en "acciones mentales", de manera que aquello que Black monitorea y controla es la decisión de Jones de hacer una determinada cosa.
} 
no intervenir si no es necesario, como de hecho ocurre, dado que Jones lleva a cabo a como resultado de su propia deliberación. Sobre este caso, la mayoría de nosotros tenemos, al menos inicialmente, la intuición de que las siguientes dos afirmaciones son verdaderas: (i) Jones no podría no haber llevado a cabo a. (ii) Jones es moralmente responsable de haber llevado a cabo $a$. Ahora bien, si (i) y (ii) son efectivamente verdaderas, tenemos un contraejemplo a PPA: un caso en el que un agente es moralmente responsable de un acto (teniendo, por tanto, el tipo de libertad que la responsabilidad moral presupone) que no podría no haber hecho. Se trata de un caso meramente posible, pero suficiente para refutar una tesis que, como PPA, se presenta como una verdad conceptual y necesaria.

Antes de proseguir, conviene plantear la siguiente pregunta: ¿cómo puede saber Black qué es lo que Jones hará? ¿cuál es la base de su juicio y de su decisión de no intervenir? Frankfurt (1969, 835) simplemente dice que Black "tiene un juicio excelente para esas cosas". Sin embargo, en discusiones posteriores de los CFs se ha intentado responder a esta pregunta introduciendo la idea de un signo previo que de manera fiable indica el comportamiento futuro de Jones y al cual Black es sensible. Supongamos, por ejemplo, que la acción $a$ que Black quiere que Jones realice es robar un coche. Y supongamos que Jones siempre se sonroja antes de robar un coche. En este caso el sonrojo de Jones es el signo previo que de manera fiable indica a Black que Jones robará el coche y que su intervención no será necesaria. Llamaremos "CF con signo previo" a los CFs que involucran este elemento y volveremos a ellos en breve.

Aunque muchos filósofos y filósofas consideran a los CFs clásicos, tal vez con ligeras variantes, como contraejemplos exitosos a PPA, también hay, como es de esperar, quienes cuestionan que las afirmaciones (i) y (ii) antes enunciadas deban darse por buenas. Una de las formas que este cuestionamiento ha adoptado es la llamada objeción del dilema, la cual está basada en la siguiente disyunción: o bien el determinismo es verdadero o bien no lo es. Esta disyunción permite construir el siguiente dilema. Primer cuerno: supongamos que el determinismo es verdadero. Entonces (ii) solo puede ser verdad si el incompatibilismo (i.e. la tesis de que el libre albedrío es incompatible con la verdad del determinismo) es falso. Y suponiendo, por mor del argumento, que el incompatibilismo no es falso, (ii) no es verdadero. (O, como alternativamente suele expresarse la conclusión de este primer cuerno del dilema, los incompatibilistas no se verán persuadidos de la verdad de (ii)). Segundo cuerno: supongamos que el determinismo es falso. Entonces, (i) no es verdad, ya que por muy fiable que sea el juicio de Black sobre el proceso deliberativo de Jones, el indeterminismo implica que siempre es posible que Jones actúe, inesperadamente, en contra del juicio de Black. Este segundo cuerno del dilema resulta 
más natural si consideramos los CFs con signo previo. Recordemos por tanto el caso antes descrito, en el cual el sonrojo de Jones es un signo fiable de que robará un coche. Dado este caso, la verdad del indeterminismo implica que no hay una conexión causal necesaria entre el sonrojo de Jones y el robo del coche (lo primero es meramente un indicador fiable de lo segundo). Pero entonces, puede perfectamente darse el caso de que Jones se sonroje, Black decida consecuentemente no intervenir, y finalmente Jones no robe el coche. Es decir, que es posible que Jones hiciera algo distinto a lo que de hecho hace. Es decir, (i) no es verdad. Vemos pues que en ambos cuernos del dilema, la conjunción de (i) y (ii) es falsa. Pero entonces no tenemos, concluye la objeción del dilema, un verdadero contraejemplo a PPA.

En su libro, Moya parece asumir que la objeción del dilema es exitosa y que obliga al defensor de los CFs a presentar versiones de estos que puedan sortearla. Este también es, al menos por mor del argumento, el punto de vista adoptado por Mele y Robb (I998), quienes presentan una versión de CF que, entre otras cosas, pretende ser inmune a la objeción del dilema. En mi opinión, por razones que no podré explicitar aquí, la objeción del dilema no es tan fuerte como puede parecerlo y no obliga realmente al defensor de CF a realizar el tipo de movimientos que Mele y Robb (entre otros) hacen para sortearla. Pero la discusión de este punto deberá quedar para otra ocasión. Aquí me ocuparé de la siguiente cuestión independiente: jes exitoso el intento de Mele y Robb de presentar un contraejemplo a PAP? (La respuesta a esta pregunta podría ser afirmativa incluso si fuera cierto que la objeción del dilema no es exitosa. En este caso, tal vez los casos tipo Mele y Robb serían innecesariamente complejos, pero no por ello fallidos.) Como he anunciado antes, Moya ofrece dos razones para pensar que Mele y Robb fracasan en su intento. Mi impresión, por contra, es que el intento es exitoso. Veamos el asunto con detenimiento.

El caso presentado por Mele y Robb (al cual en adelante llamaré "CMR"), consiste en un sujeto Bob que habita un mundo en el cual el determinismo no es verdadero (lo cual no excluye que puedan existir algunos procesos causales deterministas, solo excluye que todos los procesos causales sean deterministas). Black está interesado en que Bob robe el coche de Ann, de manera que en $\mathrm{t}_{1}$ pone en funcionamiento un proceso causal determinista $P$ que culminará en $\mathrm{t}_{2}$ con la decisión de Bob de robar el coche de Ann a menos que en $\mathrm{t}_{2}$ Bob decida robar el coche de Ann como resultado de su propia deliberación (la cual en CMR se identifica con un proceso causal indeterminista que llamaremos " $\mathrm{P}^{*}$ "). Lo que finalmente ocurre es que Bob decide robar el coche de Ann como resultado de su propia deliberación, con lo cual P no causa su decisión. Igual que Jones en los CFs clásicos, parece que Bob es responsable de su decisión (ya que esta es el resultado de su propia deliberación), pero que no 
podría haber tomado una decisión distinta a la que tomó (ya que si no hubiera decidido robar el coche de Ann como resultado de su propia deliberación, lo habría decidido como resultado de P). CMR pretende esquivar la objeción del dilema: el caso presupone explícitamente la falsedad del determinismo, pero su diseńo es tal que Bob no podría haber decidido algo distinto a lo que de hecho ha decidido. A diferencia de lo que ocurre en el CF clásico, la intervención de Black no depende del juicio que él pueda hacer a partir de la observación de la deliberación de Bob, sino que su "intervención", si podemos llamarla así, es previa e independiente a cualquier observación y a cualquier "signo previo". No existe por tanto la posibilidad de que Black "se equivoque", que decida no intervenir y que Bob no lleve a cabo la acción deseada.

\section{Moya contra la efectividad de CMR}

Moya presenta dos argumentos para la conclusión de que CMR no constituye un contraejemplo a PPA, que consideramos a continuación.

\subsection{CMR, anulación y sobredeterminación}

El primer argumento de Moya comienza por notar el proceso determinista $P$ iniciado por Black debe estar activo en el momento $t_{2}$. De lo contrario, si se desactivara antes de $t_{2}$, nada garantizaría que en $t_{2}$ Bob decidirá robar el coche, con lo cual no es verdad que Bob no podría haber decidido otra cosa en $t_{2}$. Pero, continua el argumento, "si P sigue activo en $t_{2}$, como ha de estarlo para evitar que Bob pueda decidir no robar el coche, es difícil ver cómo el proceso indeterminista podría impedir que P cause de modo determinista la decisión de Bob en $t_{2}$, por lo que el ejemplo de Mele y Robb acabaría sucumbiendo, en contra de su intención, al cuerno determinista del dilema" (116-7). Es decir, el problema parece ser que si P está activo en $t_{2}, y$ a falta de más explicaciones, deberíamos decir que P causa la decisión de Bob, en contra de la intención de Mele y Robb. ${ }^{2}$

\footnotetext{
${ }^{2}$ Un segundo problema relacionado con este, que no puedo discutir aquí, es el siguiente: tal vez la presencia del proceso determinista $\mathrm{P}$ haga que también $\mathrm{P}^{*}$ sea un proceso determinista, en contra de la intención de Mele y Robb. Entiendo que esta es la objeción de Pereboom (200I) descrita por Moya (2003). Tanto este problema, como el que discuto en el texto principal, sugieren que CMR sucumbe al cuerno determinista de la objeción del dilema. Pero por distintas razones en cada caso. En un caso, lo hace porque, en contra de la intención de Mele y Robb, P causa la decisión de Robb. En el otro, porque, también en contra de la intención de Mele y Robb, no es coherente pensar que, en presencia de $\mathrm{P}, \mathrm{P}^{*}$ sea un proceso indeterminista.
} 
Ahora bien, Mele y Robb consideran que CMR involucra lo que ellos llaman "anticipación ocurrente" (ocurrent preemption) — la cual puede ser tomada como un caso límite de la más familiar "anticipación tardía" (late preemption) o de "triunfo" (trumping) (SCHAFfer 2000)—. Es decir, según ellos, el proceso indeterminista $\mathrm{P}^{*}$ anula en $\mathrm{t}_{2}$ la eficacia causal del proceso determinista $\mathrm{P}$, de manera que aunque P siga "activo" en $t_{2}$, no es la causa de la decisión de Bob en $t_{2}$. Mele y Robb intentan mostrar que este tipo de anticipación ocurrente es coherente. Lo hacen en parte describiendo ejemplos sencillos que parecen involucrar ese tipo de anticipación (Mele y Robв 1998, 103). Si ejemplos como estos resultan coherentes, entonces también es coherente pensar que $\mathrm{P}$ sigue "activo" en $t_{2}$ sin causar la decisión de Bob en $t_{2}$.

Aunque a mí esta defensa de Mele y Robb me parece convincente, me gustaría ofrecer aquí una estrategia alternativa que ellos podrían adoptar. La estrategia consiste en mostrar que incluso si fuera cierto, en contra de lo que Mele y Robb pretenden, que P causa la decisión de Bob, el caso que ellos describen (más exactamente, el resultado de agregar a su descripción la concesión que acabamos de hacer), es igualmente un contraejemplo a PPA. Para ello, invito al lector a que imagine un caso similar a CMR, excepto por involucrar sobredeterminación causal en vez de anticipación ocurrente. Llamemos "CMR" a este nuevo caso. En $\mathrm{CMR}^{\mathrm{S}}$, tanto $\mathrm{P}$ como $\mathrm{P}^{*}$ son causas efectivas (completas e independientes) de la decisión de Bob de robar el coche de Ann. Los casos de sobredeterminación causal son inusuales, pero claramente posibles: el ejemplo típico es el de dos proyectiles disparados de manera independiente y que simultáneamente penetran en los órganos vitales de la víctima y que simultáneamente causan su muerte. El ejemplo involucra una casualidad extraordinaria, pero no una imposibilidad. A mi juicio $\mathrm{CMR}^{S}$ es un contraejemplo exitoso a PPA, aun si en $\mathrm{CMR}^{s}$ se confirma la sospecha que Moya tiene acerca de CMR: que P causa de manera determinista la decisión de Bob. Digo que es un contraejemplo exitoso a PPA por lo siguiente: (i) Bob no podría haber decidido otra cosa: dado que tanto $\mathrm{P}$ como $\mathrm{P}^{*}$ son dos causas completas e independientes de la decisión de Bob, $\mathrm{P}$ la habría causado si $\mathrm{P}^{*}$ no hubiera estado presente - es decir, si la deliberación de Bob lo hubiera inclinado hacia otra decisión-. (ii) Bob es moralmente responsable de su decisión: puede parecer que, dado que Black, a través de $\mathrm{P}$, causa la decisión de Bob, Bob queda eximido de responsabilidad moral. Pero, en mi opinión, esto no es así, porque la propia deliberación de Bob, $\mathrm{P}^{*}$, también causa su decisión. Mi impresión es que en virtud de este último hecho Bob es moralmente responsable de su decisión. Y lo es independientemente de qué haga o deje de hacer Black. Imaginemos que el robo del coche por parte de Bob ocurre exactamente igual a como ha ocurrido, excepto que Black no está presente (es decir, no se trata de un CF, 
sino de un caso de robo ordinario). En ese caso, Bob es claramente moralmente responsable de su decisión. Mi impresión es que si a este escenario simplemente "agregamos" hechos extrínsecos a los que ya están presentes (es decir, si "agregamos" a Black y el proceso P), sin hacer ninguna modificación en estos últimos, los agregados no pueden tener el efecto de disipar la responsabilidad moral de Bob. Considérese otro ejemplo: dos francotiradores A y B que actúan de manera independiente disparan balas que sobredeterminan causalmente la muerte de la víctima $\mathrm{C}$. En este caso es plausible pensar que tanto A como B son culpables del asesinato de $\mathrm{C}$, y que la culpabilidad de uno no hace desvanecer la culpabilidad del otro. De la misma manera, en $\mathrm{CMR}^{\mathrm{S}}$ la culpabilidad que debe atribuirse a Black (ya que P causa la decisión de Bob) no hace desvanecer la culpabilidad que debe atribuirse a Bob. En suma, $\mathrm{CMR}^{\mathrm{s}}$ (es decir, un caso similar a CMR excepto por involucrar sobredeterminación causal en vez de anticipación ocurrente) constituye un contraejemplo a PAP. Y lo hace a pesar de que, como Moya sospecha respecto a CMR, P cause la decisión de Bob. El hecho de que también $\mathrm{P}^{*}$ lo haga evita que $\mathrm{CMR}^{S}$ "sucumba" al cuerno determinista de la objeción del dilema. ${ }^{3}$

\subsection{CMR y sensibilidad a razones}

El segundo argumento de Moya en contra del éxito de CMR está basado en la idea de que Bob no ejerce control racional sobre su decisión (no es "sensible a razones") y que por tanto esta no puede considerarse una decisión libre, o una decisión de la cual Bob sea moralmente responsable. Consecuentemente, CMR fracasa como contraejemplo a PPA. Veamos la objeción con más detalle.

La primera premisa del argumento de Moya es que el control racional de un agente sobe su decisión o acción es una condición necesaria para que esa decisión o acción sea libre y para que podamos atribuirle al agente responsabilidad moral. ¿Qué es el control racional? Como elucidación de esta idea, Moya adopta la noción de "capacidad de dar respuesta a razones" (reasons responsiveness) elaborada por Fischer. Esta capacidad es la que ejerce un agente al tomar una decisión cuando "manteniendo constantes las estructuras de deliberación y decisión de las que hace uso en ese momento, al menos en algunas situaciones

\footnotetext{
${ }^{3}$ Quisiera notar aquí que en $\mathrm{CMR}^{S}$ queda insatisfecho el tercero de los requisitos que según Moya debe satisfacer todo CF exitoso: aquello que en un CF priva al agente de posibilidades alternativas no ha de causar su decisión y acción $(2017,103)$. En $\mathrm{CMR}^{s}$, P es a la vez lo que priva al agente de posibilidades alternativas y una causa completa de su decisión y acción. Pero, en vez de rechazar $\mathrm{CMR}^{s}$ por no satisfacer el requisito en cuestión, creo que, si procede, deberíamos modificar el requisito, tal vez en los siguientes términos: aquello que en un CF priva al agente de posibilidades alternativas no ha de ser la única causa de su decisión y acción.
} 
posibles en que hubiera razones para tomar una decisión distinta, sería sensible a estas razones y tomaría una decisión distinta" (Moya 2017, 119). El siguiente paso consiste en mostrar que en CMR Bob carece de esta capacidad de dar respuesta a razones. Para ello, Moya nos invita a considerar una variación de CMR (que en adelante llamaremos $\mathrm{CMR}^{\mathrm{R}}$ ) en la cual Bob tiene fuertes razones para no robar el coche de Ann: un amigo mecánico de Bob le asegura que el coche está equipado con un sistema anti-robo que infaliblemente lleva a la detención de cualquier persona que intente robarlo. Sin embargo, a pesar de poseer esta razón contundente para no robar el coche de Ann, Bob decide robarlo. En palabras de Moya, "cuando intenta decidir no robarlo, el mecanismo de bloqueo [es decir, el proceso determinista P] le impide decidirlo y decide robarlo de todos modos. Esta decisión es irracional y está desligada de las decisivas razones que tiene Bob para no tomarla" $(2017,121)$. Ahora bien, el hecho de que en $\mathrm{CMR}^{\mathrm{R}}$ la decisión de Bob sea irracional muestra según Moya que en CMR mismo "las estructuras de deliberación y decisión con las que Bob toma su decisión son defectuosas e incapaces de responder a razones. Debido al bloqueo, Bob no tomaría la decisión de no robar el coche de Ann aun cuando tuviera razones decisivas para tomarla" $(2017,121)$. A partir de aquí podemos concluir que en CMR Bob carece de control racional sobre su decisión, y que por tanto su decisión no es libre, y que por tanto CMR no es un contraejemplo a PPA.

Mi primera observación es que si este argumento en contra de CMR es exitoso, también lo es un argumento análogo en contra de los CFs clásicos. También sobre el CF original podría decirse que Jones carece de control racional sobre la decisión que toma, por razones análogas a las que Moya ofrece en relación a CMR. Imaginemos que Jones tiene razones contundentes para decidir hacer algo distinto a $a$. Aun en presencia de esas razones, Jones decidiría hacer $a$, ya que cuando intentara decidir algo distinto, la intervención de Black se lo impediría y terminaría decidiendo hacer $a$ en contra de sus mejores razones. Por tanto, también respecto a Jones podemos concluir que, en este caso, su decisión es irracional y desligada de las decisivas razones que tiene para no tomarla. Y análogamente a lo que ocurre en el argumento de Moya en contra de CMR, la irracionalidad de la decisión de Jones en esta variación de CF mostraría que en CF mismo Jones no es sensible a razones. Y que por tanto carece de control racional sobre su decisión. Y que por tanto su decisión no es realmente libre. Y que por tanto CF no es un contraejemplo a PPA. En suma: si el argumento de Moya en contra de CMR es exitoso, podemos construir un argumento análogo en contra de los CFs clásicos. En su libro, Moya no traza esta analogía, ni señala, creo, que la ausencia de control racional por parte de Jones sea una razón para dudar de la efectividad de los CFs clásicos. Esto puede deberse o bien a que él no crea que la analogía que he trazado sea correcta (es 
decir, que no crea que su objeción a CMR pueda extenderse a los CFs clásicos) o bien a que crea que los CFs clásicos se enfrentan a problemas más inmediatos (como la objeción del dilema) y que por tanto extender a ellos su crítica a CMR sería como llover sobre mojado.

En cualquier caso, me gustaría, en lo que queda de este trabajo, presentar de manera tentativa una razón para dudar de que la objeción de Moya a CMR (y también la objeción análoga a los CFs clásicos) sea exitosa. Mis dudas están basadas en la siguiente observación: aunque es verdad que Bob hubiera decidido en $t_{2}$ robar el coche de Ann aun en presencia de razones poderosas para no hacerlo, también es verdad que, en presencia de tales razones, Bob habría intentado decidir no robar el coche de Ann. Recuérdese que en $\mathrm{CMR}^{\mathrm{R}}$ "cuando [Bob] intenta decidir no robar [el coche de Ann], el mecanismo de bloqueo le impide decidirlo" (Moya 2017, 121). El hecho de que en presencia de buenas razones Bob intente decidir conforme a ellas (aunque finalmente no logre hacerlo) es en mi opinión significativo e indica que en algún sentido relevante Bob está siendo receptivo a razones. Es precisamente porque Bob atiende a las razones que se le presentan que él intenta decidir algo que luego, en virtud del mecanismo de bloqueo, no decide. Esto sugiere que hay al menos un tipo de capacidad de dar respuesta a razones que Bob tiene y ejerce, a la cual, y a falta de mejor nombre disponible, propongo que llamemos "capacidad básica de dar respuesta a razones". Siguiendo el ejemplo de la definición ofrecida más arriba, podemos caracterizar la capacidad básica de dar respuesta a razones como la capacidad que ejerce un agente al tomar una decisión cuando manteniendo constantes las estructuras de deliberación y decisión de las que hace uso en ese momento, al menos en algunas situaciones posibles en que hubiera razones para tomar una decisión distinta, sería sensible a estas razones e intentaría tomar una decisión distinta. ${ }^{4}$ Me parece claro que en CMR, Bob tiene esta capacidad. La cuestión siguiente (que aquí solo puedo dejar planteada) es si la capacidad básica de dar respuesta a razones es suficientemente fuerte como para ser identificada con el control racional que la libertad y la responsabilidad moral requieren. Si lo es, entonces podemos concluir que en CMR Bob toma su decisión libremente y que es moralmente responsable de haberla tomado. Y, por tanto, que CMR es un contraejemplo a PPA.

\footnotetext{
${ }^{4}$ Para no extenderme más de la cuenta, estoy tomando aquí a los intentos (contrafácticos) del agente por actuar de acuerdo a razones como constitutivos de la capacidad básica de dar respuesta a razones. No obstante, también me parece plausible que tales intentos sean simplemente un sintoma, que en algunos casos emerge, de otros hechos más básicos, y que sean esos otros hechos más básicos a los que haya que aludir en análisis correcto de la capacidad básica de dar respuesta a razones. Es decir, aunque creo que la caracterización aquí ofrecida de la capacidad básica de dar respuesta a razones es útil para los fines presentes, me parece plausible que tal caracterización no sea el análisis último de la noción.
} 
Aunque, como digo, no puedo responder aquí la pregunta de si la capacidad básica de dar respuesta a razones es suficientemente fuerte como para ser identificada con el control racional que la libertad y la responsabilidad moral requieren, sí quisiera anticiparme a una posible respuesta negativa. Alguien podría argumentar de la siguiente manera:

Todos estamos de acuerdo en que en $\mathrm{CMR}^{\mathrm{R}}$ (a) la acción de Bob no es libre. También deberíamos estar de acuerdo en que (b) hay un tipo de control racional que está ausente: la decisión de Bob, al fin y al cabo, es irracional, contraria a las razones que Bob ha considerado. Sin embargo, (c) Bob tiene la capacidad básica de responder a razones: Bob ha considerado sus razones y ha intentado actuar conforme a ellas. Pero entonces, en vistas de (a), (b) y (c), deberíamos concluir que la capacidad básica de responder a razones (que está presente en $\mathrm{CMR}^{\mathrm{R}}$ ) no es suficiente para capturar el tipo de control racional requerido por la libertad y la responsabilidad moral (que están ausentes en $\mathrm{CMR}^{\mathrm{R}}$ )

Este argumento es en mi opinión incorrecto, por lo siguiente: la razón por la que juzgamos que en $\mathrm{CMR}^{\mathrm{R}}$ la decisión no es libre no tiene por qué ser la ausencia de control racional en un sentido más fuerte que el de la capacidad básica de dar respuesta a razones. La razón puede perfectamente ser que en $\mathrm{CMR}^{\mathrm{R}}$ la decisión de Bob es fruto de la intervención de Black y que por tanto Bob no es autor genuino de su decisión. Queda por tanto abierta la cuestión de si el tipo de control racional del que Bob carece en $\mathrm{CMR}^{\mathrm{R}}$ es realmente necesario para la libertad y la responsabilidad moral, o si más bien lo que es necesario es el tipo de control racional que Bob sí posee (es decir, la capacidad básica de responder a razones). Para establecer lo primero sería necesario describir un caso en el que además de satisfacerse las condiciones (a), (b) y (c) también se satisfagan todas las condiciones necesarias para la libertad y responsabilidad moral que no sean objeto de discusión —algo que no ocurre en $\mathrm{CMR}^{\mathrm{R}}$ - .

\section{Conclusión}

En este artículo he intentado defender los casos tipo Mele/Robb (CMR) de los dos argumentos que en contra de su efectividad ofrece Carlos Moya en su libro El libre albedrío. El primer argumento de Moya está basado en la sospecha de que en CMR el mecanismo de bloqueo termina siendo causa de la acción del agente, en contra de las intenciones de Mele y Robb. Mi respuesta ha consistido en mostrar que aun cuando esa sospecha sea cierta, el caso descrito por Mele y Robb seguiría siendo efectivo (aunque de una manera ligeramente 
distinta a la que ellos pretenden). El segundo argumento de Moya está basado en la idea de que en CMR el agente carece de control racional y que por tanto no es realmente libre. Mi línea central de respuesta a este segundo argumento ha sido la de mostrar que hay un tipo de control racional que el agente en CMR sí posee y que tal vez ese tipo de control racional sea todo el control racional que la libertad y la responsabilidad moral necesitan. Si mis respuestas a los argumentos de Moya son correctas, entonces parece que CMR sigue siendo un contrajemplo genuino a PPA, y que por tanto el control plural no es una condición necesaria para la libertad y la responsabilidad moral. 


\section{BibLIOGRAFÍA}

Frankfurt, H. G. I969, "Alternate Possibilities and Moral Responsibility", Journal of Philosophy, 66: 829.

Mele, A. R. y Roвb, D. I998, "Rescuing Frankfurt-style cases", Philosophical Review, 107: 97-112.

Moya, C. 2003, "Blockage cases. No case against PAP", Crítica. Revista Hispanoamericana de Filosofía, 35, 104: 109-20

Moya, C. 2017, El libre albedrio. Un estudio filosófico, Cátedra.

Pereboom, D. 200 i, Living Without Free Will, Cambridge University Press.

Schaffer, J. 2000, “Trumping Preemption”, Journal of Philosophy, 9: 165-81. 